\title{
Estimation of formamide harmonic and anharmonic modes in the Kohn-Sham limit using the polarization consistent basis sets
}

\author{
Aneta Buczek • Teobald Kupka • Malgorzata A. Broda
}

Received: 15 September 2010 / Accepted: 23 December 2010 /Published online: 26 January 2011

(C) The Author(s) 2011. This article is published with open access at SpringerLink.com

\begin{abstract}
Formamide harmonic and anharmonic frequencies of fundamental vibrations in the gas phase and in several solvents were successfully estimated in the B3LYP KohnSham complete basis set limit (KS CBS). CBS results were obtained by extrapolating a power function (two-parameter formula) to the results calculated with polarization-consistent basis sets. Anharmonic corrections using the second order perturbation treatment (PT2) and hybrid B3LYP functional combined with polarization consistent pc- $n(n=0,1,2,3,4)$ and several Pople's basis sets were analyzed for all fundamental formamide vibrational modes in the gas phase and solution. Solvent effects were modeled within a PCM method. The anharmonic frequency of diagnostic amide vibration $\mathrm{C}=\mathrm{O}$ in the gas phase and the $\mathrm{CCl}_{4}$ solution calculated with the VPT2 method was significantly closer to experimental data than the corresponding harmonic frequency. Both harmonic and anharmonic frequencies of $\mathrm{C}=\mathrm{O}$ stretching mode decreased linearly with solvent polarity, expressed by relative environment permittivity $(\varepsilon)$ ratio $(\varepsilon-$ $1) /(2 \varepsilon+1)$. However, an unphysical behavior of solvent dependence of some low frequency anharmonic amide modes of formamide (e.g., $\mathrm{CN}$ stretch, $\mathrm{NH}_{2}$ scissoring, and $\mathrm{NH}_{2}$ in plane bend) was observed, probably due to the presence of severe anharmonicity and Fermi resonance.
\end{abstract}

Keywords Harmonic vibration - Anharmonic vibration . Complete basis set limit $\cdot$ Formamide $\cdot$ Solvent effect

Electronic supplementary material The online version of this article (doi:10.1007/s00894-010-0944-9) contains supplementary material, which is available to authorized users.

A. Buczek $\cdot$ T. Kupka $\cdot$ M. A. Broda $(\bowtie)$

Faculty of Chemistry, University of Opole,

48 Oleska Street,

45-052 Opole, Poland

e-mail: broda@uni.opole.pl

\section{Introduction}

IR and Raman vibrational spectroscopies are used widely for characterization of both novel chemical compounds and natural products. Calculated harmonic frequencies are usually overestimated due to neglect of anharmonicity effects [1]. For example, the high frequency wavelengths calculated by Hartree-Fock method are typically overestimated by about $10 \%$. Although scaling of harmonic frequencies computed at the Hartree-Fock and other levels of theory gives reasonable results, the development of hardware and software allow the a priori prediction of anharmonic wavenumbers. There are three fundamental studies [2-4] on scaling factors used in frequency and ZPV calculations. Evaluation of scaling factors is very laborious work and, therefore, despite the presence of myriad methods and basis sets, only a few scaling factors are available in the literature. In particular, scaling of results obtained with the recently introduced Jensen's basis sets [5-10] and very large Dunning's [11-14] basis sets are lacking. Moreover, in more accurate theoretical vibrational studies, the effect of solvent, present in most experimental works should be also included.

Among the high number of basis sets available, the socalled Pople basis sets (see ref. [1]), though fairly old, are robust and relatively small. Sometimes, they reproduce very well the experimental parameters of medium-sized molecules. However, no regular change of atomic and molecular energy is calculated with the Pople basis sets toward the complete basis set limit (CBS). Dunning and coworkers [11-14] utilized the idea of smooth and regular converging energy toward the CBS for constructing correlationconsistent basis set hierarchies. Thus, in theoretical thermochemical calculations, the energy, and some other structural and spectral parameters, have been estimated 
accurately using simple 2- and 3-parameter formulae [12, 15-17]. Later, Jensen [5-10] designed another family of converging basis sets, the so-called polarized-consistent basis sets pc-n, where $n=0,1,2,3$ and 4 . These basis set series, developed explicitly for DFT, seem to converge faster than those of Dunning while reproducing the parameters calculated with the latter in the corresponding SCF, DFT, MP2 and $\operatorname{CCSD}(\mathrm{T})$ basis set limits [18]. Despite the fact that the use of the CBS approach in DFT methods can be controversial, several studies have shown regular convergence of molecular energy and other parameters toward the corresponding Kohn-Sham (KS) limit $[19,20]$.

Formamide is the simplest model of a peptide bond and is often used for model experimental and theoretical studies related to protein chemistry. Cappelli [22] reported anharmonic frequencies of selected formamide modes in the gas phase and in water solution calculated by B3LYP/6-311+ + $\mathrm{G}^{* *}$ method. There have been several detailed and highlevel studies on anharmonicity of formamide modes [2325]. However, to the best of our knowledge, there have been no studies on formamide harmonic and anharmonic frequencies convergence toward the KS limit using a hierarchy of systematically changed basis sets and the influence of solvent on the obtained results. Do harmonic and anharmonic vibrations of formamide calculated with pc-n basis set hierarchy monotonically converge toward the KS limit? This question would be of interest for future detailed studies of vibrational problems. Boese and coworkers [21] used Dunning's and pc-1, 2 and 3 basis sets, and reported on convergence of anharmonic vibrations in case of 17 small molecules but formamide was not considered. Besides, no CBS estimates were reported in their paper.

In this work, we report on the convergence of harmonic and anharmonic fundamental frequencies of formamide modes in the gas phase and solution, calculated with second-order vibrational perturbation theory (VPT2) $[26,27]$ and pc-n polarization-consistent basis sets toward the B3LYP CBS. The results of individual calculations using a hierarchy of pc-n basis sets were fitted using a simple two-parameter mathematical formula. For comparison, calculations using several Pople basis sets were performed. The results obtained with individual basis sets and the estimated CBS values were critically compared with available experimental data in the gas phase [28] and solution [29].

\section{Computational details}

All calculations were performed using the Gaussian 09 program [30].
Basis sets and density functionals

Pople's 3-21G, 6-31G, 6-31G*, 6-31+G**, 6-311++G** and 6$311++\mathrm{G}(3 \mathrm{df}, 2 \mathrm{pd})$ (see details in ref. [30] and references therein), and Jensen's pc-n polarized-consistent basis sets [5-10] were used. Efficient B3LYP density functional was selected. The pc$\mathrm{n}$ basis sets were downloaded from EMSL [31].

\section{Geometry}

Fully optimized geometries of formamide in the gas phase, and solution (with PCM model [32]) were obtained using default and tight convergence criteria for each method and basis set selected. All positive harmonic vibration frequencies were obtained ensuring ground state structures.

Harmonic and anharmonic vibration calculations

The calculations were carried out in the gas phase and for eight selected solvents (n-hexane, chloroform, carbon tetrachloride, acetone, acetonitrile, DMSO, water and formamide) using the VPT2 method as implemented by Barone $[26,27]$ in the Gaussian program package [30]. In all cases, the finest DFT integration grid was selected by using SCF=tight and $\operatorname{Int}($ Grid=150590) in the command line instead of Int(Grid=ULTRAFINE) keyword. The use of such a fine grid is critical in the case of anharmonic frequency calculations with large basis sets [33]. Fermi resonances were handled in all calculations by default settings in the G09 anharmonic calculations.

\section{CBS calculations}

The harmonic and anharmonic frequencies, $Y(X)$, were calculated using polarization-consistent $\mathrm{pc}-\mathrm{n}$ basis sets, where $n=0,1,2,3$ and 4 , and subsequently extrapolated to the B3LYP CBS limit, $Y(\infty)$, by fitting the individual results to the two-parameter function [15]:

$Y(\mathrm{X})=Y(\infty)+\mathrm{A} / \mathrm{X}^{3}$

The extrapolated value $Y(\infty)$ corresponds to the best estimate of the predicted frequency for infinite zeta (or cardinal number " $X$ "), and $A$ and $Y(\infty)$ are fitted parameters. In case of Jensen's pc-n basis sets, $X=n+2$, was assumed for graphical fitting purposes only [18, 34]. All the fittings were performed with two-parameter formula (Eq. 1). Since smaller values of " $\mathrm{X}$ " and " $\mathrm{n}$ " yield results (frequencies in this study) more corrupted by errors due to basis set imperfections, the CBS values are often estimated using higher cardinal numbers. For example, the abbreviation CBS $(4,5,6)$ indicates estimation using $\mathrm{X}=\mathrm{Q}, 5$ and 6 , or $\mathrm{n}=$ 2, 3 and 4 , respectively. 
Scaling factors

Single scaling factors were used for low and high frequencies. Thus, in several cases we arbitrary took the values from partly similar basis sets [2-4]. For the reader's convenience, all the scaling factors used in our work are collected in one table (Table S4 in the supporting material).

\section{Results and discussion}

Figure 1 shows B3LYP calculated harmonic and anharmonic frequencies of six selected (amidic) formamide modes in the gas phase as a function of selected Pople and Jensen basis set size. Experimental values [28] are added as solid straight lines and the results obtained with pc-2, pc-3 and pc-4 basis sets are nonlinearly fitted toward the CBS using Eq. 1. The corresponding CBS values for harmonic and anharmonic frequencies are shown as straight dashed lines. Most results obtained with Jensen's basis set (starting from pc-2) converge smoothly toward the B3LYP KS limit. On the other hand, both harmonic and anharmonic frequencies obtained with selected Pople type basis set show some irregular dependence on basis set quality but the values obtained with the largest $[6-311++\mathrm{G}(3 \mathrm{df}, 2 \mathrm{pd})]$ are close to those estimated in the CBS limit. CBS values of harmonic and anharmonic frequencies of asymmetric and symmetric $\mathrm{NH}_{2}$ stretching modes behave typically, e.g., the anharmonic ones are lower by about 200 and $170 \mathrm{~cm}^{-1}$, respectively, and are closer to experimental values [28]. The excellent agreement with experiment is visible for anharmonic CBS values of carbonyl stretching and $\mathrm{NH}_{2}$ scissoring modes. However, in the VPT2 method it is more challenging to take into account the anharmonicity of low frequency modes. Thus, for example, the harmonic frequencies of $\mathrm{CN}$ stretch predicted with both Pople and Jensen's basis sets are close to experimental values and show very little dependence on basis set quality. On the contrary, the corresponding anharmonic frequencies for pc$\mathrm{n}$ basis sets regularly (and significantly) decrease toward the CBS. This leads to the highest absolute deviation in the CBS $\left(-117 \mathrm{~cm}^{-1}\right)$, significantly larger than that produced with smaller basis sets. The reason for such a large dependence on basis set size is probably the Fermi resonance between this mode and the first overtone of $\mathrm{NCO}$ bending. Surprisingly, in the case of in plane $\mathrm{NH}_{2}$ bending, the anharmonic frequencies calculated with the 6$31 \mathrm{G}^{*}$ and $6-31+\mathrm{G}^{* *}$ basis sets are higher than the corresponding harmonic ones. It difficult to explain this result since this mode does not participate in Fermi resonance with any other vibrational mode.

Table 1 gathers the deviations from experimental values of all harmonic and anharmonic frequencies of formamide in the gas phase calculated using the B3LYP hybrid density functional and selected Pople and Jensen basis sets. Results obtained with several additional basis sets are collected in Table S1 in the electronic supplementary material. The accuracy of frequency prediction depends strongly on the mode type. In most cases, the deviations from experimental values of anharmonic frequencies are smaller as compared to those of harmonic frequencies [28]. Moreover, the anharmonic frequency of the $\mathrm{C}=\mathrm{O}$ stretch is predicted accurately, while the $\mathrm{NH}_{2}$ wagging is very erratic for all basis sets used. In particular, for the $6-31 \mathrm{G}^{*}$ basis set an abnormal change of frequency deviation for this mode from $-207\left(\Delta_{\text {harm }}\right)$ to $2245 \mathrm{~cm}^{-1}\left(\Delta_{\text {anharm }}\right)$ is observed. This results in a very large root mean square (RMS) for this particular basis set $\left(650 \mathrm{~cm}^{-1}\right)$. When the deviations for this mode are excluded, the corresponding RMS values are significantly smaller (see Table 1). The inability of the VPT2 method to properly treat formamide $\mathrm{NH}_{2}$ wagging (and other modes that exhibit large anharmonicity and strong resonances) in comparison to other methods, like VSCF or VCI, was already reported by Bounouar [23]. Earlier, Bour [24] observed poor agreement with experimental values in the case of six lower frequency anharmonic modes calculated by the VPT2 method. Among Pople's basis set, the smallest RMS for anharmonic vibrations was observed for the $6-31+\mathrm{G}^{* *}$ and $6-311++$ $\mathrm{G}^{* *}$ basis sets, and the largest used basis set produced a significantly poorer result (RMS of 30,34 and $46 \mathrm{~cm}^{-1}$ ). Similarly, RMS deviation of anharmonic frequencies calculated for the pc-2 basis set is smaller than estimated for the CBS limit (42 vs $59 \mathrm{~cm}^{-1}$ ). On the other hand, the use of a single scaling factor (the scaling factors used are gathered in Table S4 of the supplementary material) results in deviations significantly smaller than those observed for anharmonic frequencies [28].

The performance of the VPT2 method depends also on the method of calculation. For example, the MP2/aug-ccpVTZ calculated anharmonic $\mathrm{NH}_{2}$ stretch modes [23] reproduced the experimental data ideally (by about 2 $\mathrm{cm}^{-1}$ ) while our B3LYP results with both Pople and Jensen's basis sets show large discrepancies (about 30-50 $\mathrm{cm}^{-1}$ ). However, both methods predict very well the CNO bending mode, probably due to the very low degree of anharmonicity.

Next, the performance of VPT2 in predicting anharmonic formamide frequencies in solution was studied using the PCM approach. Both low and high polarity solvents were tested. First, the impact of low polarity solvent $\left(\mathrm{CCl}_{4}\right)$ on anharmonic vibrations for the studied Pople and Jensen basis sets was checked; a picture very similar to that presented in Fig. 1 was obtained (see Fig. S1 in the supplementary material). Similarly to formamide in the gas phase, the CBS values of anharmonic frequencies of two 
Fig. 1 Sensitivity of B3LYPcalculated selected harmonic and anharmonic frequencies of formamide in the gas phase to selected Pople and polarization consistent basis sets quality. The results for pc-n basis sets were fitted with Eq. 1 and the complete basis set limit (CBS) $(2,3,4)$ estimated
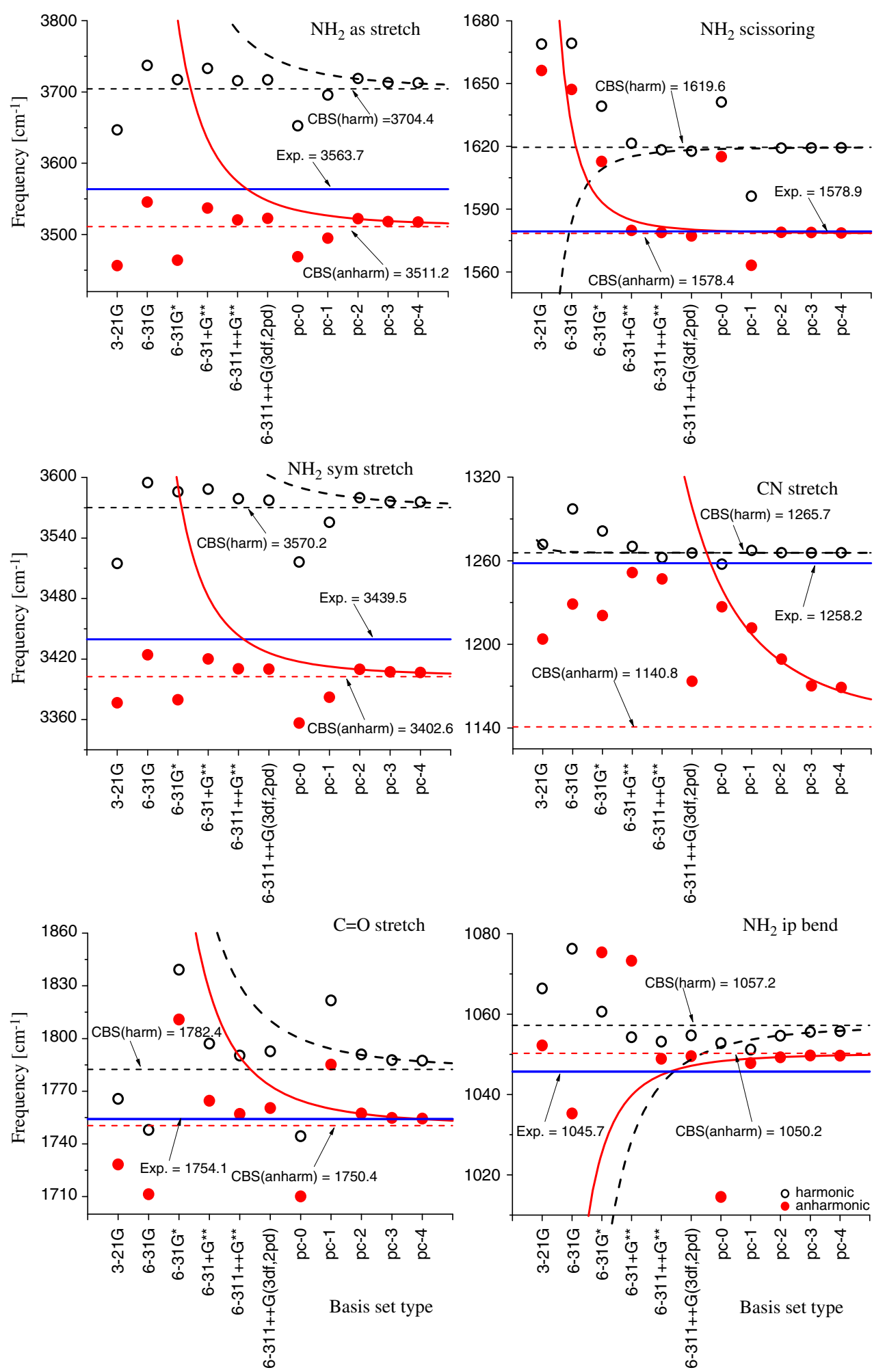

$\mathrm{NH}_{2}$ and $\mathrm{C}=\mathrm{O}$ stretch modes reproduced very well the available experimental wavenumbers (the corresponding deviations are $-30,-19$ and $-6 \mathrm{~cm}^{-1}$ [29]). However, the anharmonic frequency of $\mathrm{C}-\mathrm{N}$ stretching is very sensitive to the basis set quality and its $\mathrm{CBS}$ value underestimates the experimental value in $\mathrm{CCl}_{4}$ solution significantly (by $-102 \mathrm{~cm}^{-1}$ ) [29]. On the contrary, the calculated harmonic $\mathrm{CN}$ stretching mode is almost independent of the basis set size and quality, and overestimates the corresponding experimental values by only $23 \mathrm{~cm}^{-1}$.

Table 2 presents all formamide harmonic and anharmonic frequencies in $\mathrm{CCl}_{4}$ solution calculated using 
Table 1 Deviations from experimental values of formamide harmonic $\left(\Delta_{\text {harm }}\right)$, anharmonic $\left(\Delta_{\text {anharm }}\right)$ and scaled harmonic $\left.\Delta_{\text {scal }}\right)$ frequencies $\left(\mathrm{cm}^{-1}\right)$ calculated with B3LYP and selected Pople and Jensen's basis sets. RMS Root mean square

\begin{tabular}{|c|c|c|c|c|c|c|c|c|c|c|c|c|c|c|}
\hline \multirow[b]{2}{*}{ No. } & \multirow[b]{2}{*}{ mode } & \multirow[b]{2}{*}{$\exp ^{\text {a }}$} & \multicolumn{3}{|c|}{$6-31 \mathrm{G}^{*}$} & \multicolumn{3}{|c|}{$6-311++\mathrm{G}(3 \mathrm{df}, 2 \mathrm{pd})$} & \multicolumn{3}{|l|}{ pc-2 } & \multicolumn{3}{|l|}{ CBS } \\
\hline & & & $\Delta_{\text {harm }}$ & $\Delta_{\text {anharm }}$ & $\Delta_{\text {scal }}$ & $\Delta_{\text {harm }}$ & $\Delta_{\text {anharm }}$ & $\Delta_{\text {scal }}$ & $\Delta_{\text {harm }}$ & $\Delta_{\text {anharm }}$ & $\Delta_{\text {scal }}$ & $\Delta_{\text {harm }}$ & $\Delta_{\text {anharm }}$ & $\Delta_{\text {scal }}$ \\
\hline 1 & $\mathrm{NH}_{2}$ as stretch & 3564 & 154 & -100 & 10 & 153 & -41 & 37 & 155 & -41 & 40 & 141 & -53 & 48 \\
\hline 2 & $\mathrm{NH}_{2}$ sym stretch & 3440 & 146 & -60 & 8 & 138 & -30 & 25 & 140 & -29 & 30 & 131 & -37 & 42 \\
\hline 3 & $\mathrm{CH}$ stretch & 2854 & 110 & -57 & -5 & 88 & -90 & -5 & 91 & -89 & 0 & 80 & -93 & 7 \\
\hline 4 & $\mathrm{C}=\mathrm{O}$ stretch & 1754 & 85 & 57 & 14 & 39 & 6 & -18 & 37 & 4 & -19 & 28 & -4 & -16 \\
\hline 5 & $\mathrm{NH}_{2}$ scissoring & 1579 & 60 & 34 & -3 & 39 & -2 & -12 & 40 & 0 & -10 & 41 & -1 & 0 \\
\hline 6 & $\mathrm{CH}$ bend & 1391 & 47 & 57 & -9 & 30 & 33 & -15 & 31 & 33 & -13 & 29 & 33 & -7 \\
\hline 7 & $\mathrm{CN}$ stretch & 1258 & 23 & -37 & -26 & 7 & -85 & -32 & 7 & -67 & -32 & 7 & -117 & -24 \\
\hline 8 & $\mathrm{NH}_{2}$ ip bend & 1046 & 15 & 30 & -26 & 9 & 4 & -24 & 9 & 4 & -24 & 12 & 5 & -15 \\
\hline 9 & $\mathrm{CH}$ op bend & 1033 & 12 & -20 & -28 & 9 & -13 & -23 & 11 & -11 & -21 & 8 & -18 & -18 \\
\hline 10 & $\mathrm{NH}_{2}$ tors & 602 & 49 & -60 & 24 & 37 & -65 & 16 & 39 & -50 & 19 & 33 & -99 & 18 \\
\hline 11 & NCO bend & 566 & -2 & 3 & -23 & 4 & 5 & -14 & 3 & 5 & -14 & 7 & 1 & -8 \\
\hline \multirow[t]{3}{*}{12} & $\mathrm{NH}_{2}$ wag & 289 & -207 & 2245 & -210 & -42 & 157 & -50 & -34 & 156 & -42 & -40 & 144 & -46 \\
\hline & RMS & & 98 & 650 & 63 & 69 & 63 & 26 & 70 & 60 & 25 & 64 & 70 & 26 \\
\hline & $\mathrm{RMS}^{\mathrm{b}}$ & & 82 & 53 & 19 & 71 & 46 & 22 & 72 & 42 & 23 & 66 & 59 & 23 \\
\hline
\end{tabular}

${ }^{\text {a }}$ Reference [28]

${ }^{\mathrm{b}}$ RMS calculated without $\mathrm{NH}_{2}$ wagging mode

selected Pople and Jensen basis sets; the complete set of results is shown in the electronic supplementary material (Table S2). First, a very nice reproduction of experimental $\mathrm{C}=\mathrm{O}$ frequency [29] by the VPT2 method is visible for better quality basis sets $\left[6-31+\mathrm{G}^{* *}, 6-311++\mathrm{G}^{* *}, 6-311\right.$
$++\mathrm{G}(3 \mathrm{df}, 2 \mathrm{pd})$ and $p c-n$, for $n=2-4]$ is apparent. On the contrary, the $\mathrm{NH}_{2}$ wagging frequency (observed at 323 $\mathrm{cm}^{-1}$ ) is correctly predicted by a simple harmonic model but significantly overestimated by VPT2 method $\left(1,186 \mathrm{~cm}^{-1}\right.$ for $6-31 \mathrm{G}^{*}$ basis set and about $500 \mathrm{~cm}^{-1}$ for $6-311++\mathrm{G}$

Table 2 Deviations ${ }^{\mathrm{a}}$ of formamide harmonic $\left(\Delta_{\text {harm }}\right)$ and anharmonic $\left(\Delta_{\text {anharm }}\right)$ frequencies $\left(\mathrm{cm}^{-1}\right)$ in $\mathrm{CCl}_{4}$ solution calculated by B3LYP with selected Pople and Jensen's basis sets. $C B S$ Complete basis set limit

\begin{tabular}{|c|c|c|c|c|c|c|c|c|c|c|}
\hline \multirow[b]{2}{*}{ No. } & \multirow[b]{2}{*}{ Mode } & \multirow[b]{2}{*}{ exp. ${ }^{b}$} & \multicolumn{2}{|c|}{$6-31 \mathrm{G}^{*}$} & \multicolumn{2}{|c|}{$6-311++\mathrm{G}(3 \mathrm{df}, 2 \mathrm{pd})$} & \multicolumn{2}{|l|}{ pc-2 } & \multicolumn{2}{|l|}{ CBS } \\
\hline & & & $\Delta_{\text {harm }}$ & $\Delta_{\text {anharm }}$ & $\Delta_{\text {harm }}$ & $\Delta_{\text {anharm }}$ & $\Delta_{\text {harm }}$ & $\Delta_{\text {anharm }}$ & $\Delta_{\text {harm }}$ & $\Delta_{\text {anharm }}$ \\
\hline 1 & $\mathrm{NH}_{2}$ as stretch & 3534 & 179 & -41 & 177 & -23 & 179 & -23 & 164 & -30 \\
\hline 2 & $\mathrm{NH}_{2}$ sym stretch & 3411 & 173 & -15 & 163 & -14 & 166 & -14 & 156 & -19 \\
\hline 3 & $\mathrm{CH}$ stretch & 2862 & 117 & -74 & 100 & -96 & 103 & -97 & 92 & -93 \\
\hline 4 & $\mathrm{C}=\mathrm{O}$ stretch & 1721 & 95 & 61 & 40 & 4 & 39 & 2 & 31 & -6 \\
\hline 5 & $\mathrm{NH}_{2}$ scissoring & - & - & - & - & - & - & - & - & - \\
\hline 6 & $\mathrm{CH}$ bend & 1391 & 49 & 43 & 33 & 23 & 34 & 22 & 32 & 29 \\
\hline 7 & $\mathrm{CN}$ stretch & 1252 & 36 & -36 & 23 & -84 & 23 & -77 & 23 & -102 \\
\hline 8 & $\mathrm{NH}_{2}$ ip bend & 1216 & -148 & -143 & -153 & -226 & -153 & -225 & -150 & -220 \\
\hline 9 & $\mathrm{CH}$ op bend & 1064 & -12 & -43 & -13 & -37 & -12 & -36 & -15 & -34 \\
\hline 10 & $\mathrm{NH}_{2}$ tors & 667 & -11 & -28 & -20 & -59 & -18 & -50 & -23 & -76 \\
\hline 11 & NCO bend & 573 & -5 & -3 & 1 & -55 & 0 & -58 & 3 & -46 \\
\hline \multirow[t]{3}{*}{12} & $\mathrm{NH}_{2}$ wag & 323 & -167 & 863 & -26 & 202 & -21 & 200 & -28 & 224 \\
\hline & $\mathrm{RMS}^{\mathrm{c}}$ & & 112 & 267 & 93 & 103 & 94 & 102 & 89 & 108 \\
\hline & $\mathrm{RMS}^{\mathrm{d}}$ & & 105 & 61 & 97 & 88 & 98 & 86 & 93 & 89 \\
\hline
\end{tabular}

${ }^{\text {a }} \Delta=v_{\text {calc }}-v_{\exp }$

${ }^{b}$ Reference [29]

c $\mathrm{RMS}$ calculated without $\mathrm{NH}_{2}$ scissoring mode

d RMS calculated without $\mathrm{NH}_{2}$ scissoring and $\mathrm{NH}_{2}$ wagging modes 
(3df,2pd) and pc-n, for $n=2-4$ ). The best overall result for the VPT2 method is observed for the 6-31G* basis set (RMS of 105 and $61 \mathrm{~cm}^{-1}$ for harmonic and anharmonic frequencies), and there is no accuracy gain when using pc$\mathrm{n}$ basis sets (RMS of 98 and $86 \mathrm{~cm}^{-1}$ for harmonic and anharmonic frequencies calculated using pc-2 basis set, and 93 vs $89 \mathrm{~cm}^{-1}$ in the CBS limit). The apparently best result for the $6-31 \mathrm{G}^{*}$ basis set is, however, due to incidental error cancellation since the corresponding results for $6-311++\mathrm{G}$ (3df,2pd) basis set are similar to those obtained in the complete basis set limit (see Table 2). Surprisingly, the largest deviation in $\mathrm{CCl}_{4}$ (about $-200 \mathrm{~cm}^{-1}$ ) is observed for anharmonic $\mathrm{NH}_{2}$ in plane bending. On the contrary, this mode is very well predicted in the gas phase (deviation of about $5 \mathrm{~cm}^{-1}$ ). However, comparing the experimental wavenumbers, assigned in the gas phase and solution (1046 vs 1216 $\mathrm{cm}^{-1}$ ), one could question the latter assignment. The overall RMS values for both harmonic and anharmonic frequencies in $\mathrm{CCl}_{4}$ solution are significantly larger than the corresponding numbers in the gas phase. Thus, the results from Table 2 show no advantage of using the VPT2 approach to calculate accurate frequencies of formamide in $\mathrm{CCl}_{4}$ solution ( $\mathrm{C}=\mathrm{O}$ mode is a striking exception to this rule).

There are no reported experimental data on all the different formamide vibrational modes in various solvents and only some sparse data exist [29]. This is partly due to problems caused by formamide association. Therefore, Table 3 compares harmonic and anharmonic frequencies calculated at B3LYP/pc-2 level of theory for selected vibrational modes of formamide in vacuum and in eight solvents. It is apparent from Table 3 that, both for the isolated molecule and in solution, the anharmonic frequency of the $\mathrm{C}=\mathrm{O}$ mode is lower than the harmonic frequency. Moreover, upon using more polar solvent, the harmonic and anharmonic frequency of this vibration decreases in a roughly linearly manner. However, the changes in frequency introduced by solvent are more complex for other vibrational modes. In the case of the three amide modes $\left(\mathrm{NH}_{2}\right.$ scissoring, $\mathrm{NH}_{2}$ in plane bend and $\mathrm{NCO}$ bending modes) an unphysical positive anharmonicity is observed for more polar solvents. A particularly high positive anharmonicity $\left(\sim 200 \mathrm{~cm}^{-1}\right)$ in all solvents is calculated for $\mathrm{NH}_{2}$ wagging mode. In fact, four cases of Fermi resonances were calculated in the gas phase and solvents of low polarity, and seven Fermi resonances in more polar solvents. For example, $\mathrm{NH}_{2}$ scissoring mode $(v 5)$ is in Fermi resonance with modes $v 9+v 8$. Quite a similar pictures was obtained from the corresponding B3LYP/6$31+\mathrm{G}^{* *}$ calculations (for brevity these results are presented in Table S4 in the electronic supplementary material). Thus, Fermi resonance could be responsible for the observed unphysical behavior of several anharmonic modes calculated in polar solvents.

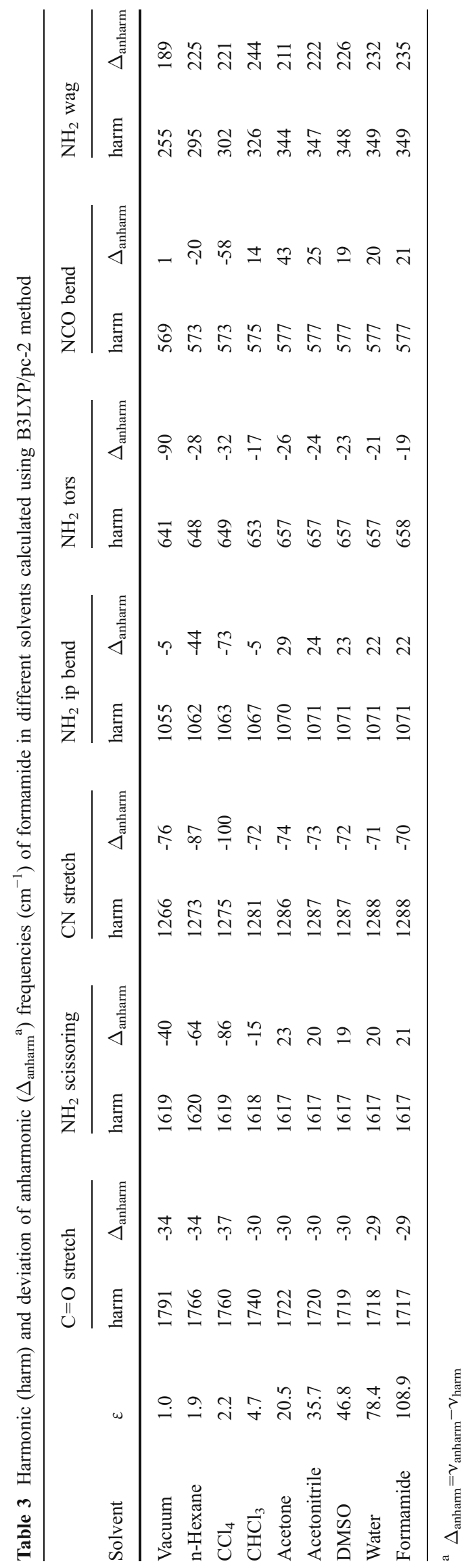


Fig. 2 Solvent dependence of selected formamide harmonic and anharmonic frequencies calculated at the B3LYP/pc-2 level
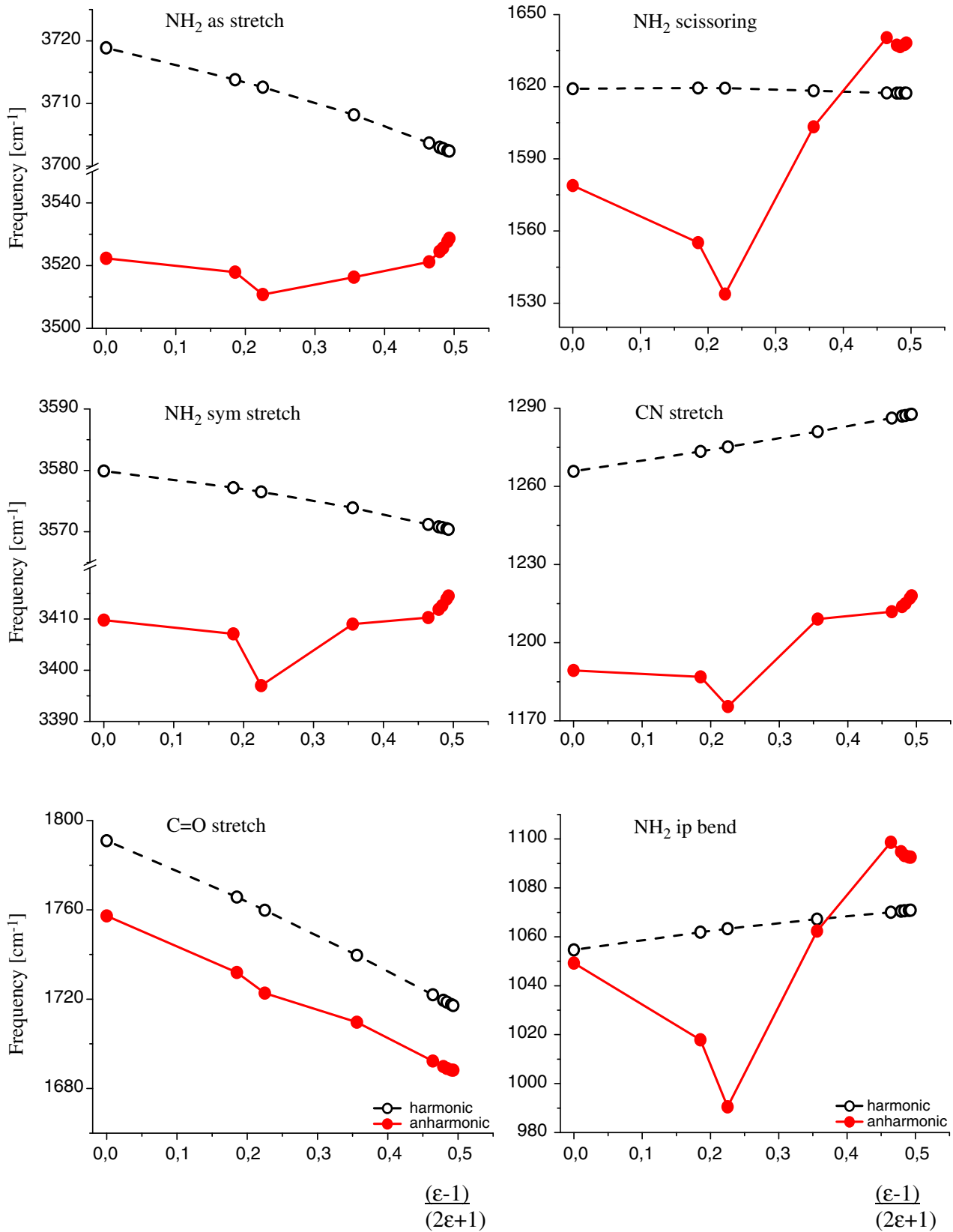

Figure 2 presents selected formamide harmonic and anharmonic frequencies, calculated at the B3LYP/pc-2 level of theory as a function of medium polarity, expressed as $(\varepsilon-1) /(2 \varepsilon+1)$. The regular changes in harmonic frequencies are roughly linear and agree with our chemical intuition. However, the corresponding anharmonic frequencies show some abrupt and unexpected behavior (there is a kind of more or less pronounced minimum for $\mathrm{CCl}_{4}$ ). Moreover, anharmonic frequencies of $\mathrm{NH}_{2}$ scissoring and $\mathrm{NH}_{2}$ in plane bending in more polar solvents are higher than the corresponding harmonic frequencies. A very similar result is produced by $\mathrm{B} 3 \mathrm{LYP} / 6-31+\mathrm{G}^{* *}$ calculations (see Fig. S2 in the supplementary material). This is clearly some kind of artifact of handling anharmonicity in solution, possibly introduced by the VPT2 method. To the best of our knowledge, this artificial behavior of anharmonic frequencies upon solvent polarity change has not been reported before.

Looking for a possible explanation for the poor behavior of anharmonic frequencies of formamide in solution, we considered two reasons: limitations of the PCM model or VPT2 method, and/or its implementation for studies in solution. Intuitively, the correct behavior of the PCM model in solvents of increased polarity should results in linear changes of structural parameters optimized for both equilibrium and rovibrationally averaged geometry. We thus analyzed the corresponding changes introduced by solvent to selected geometrical parameters of formamide. 


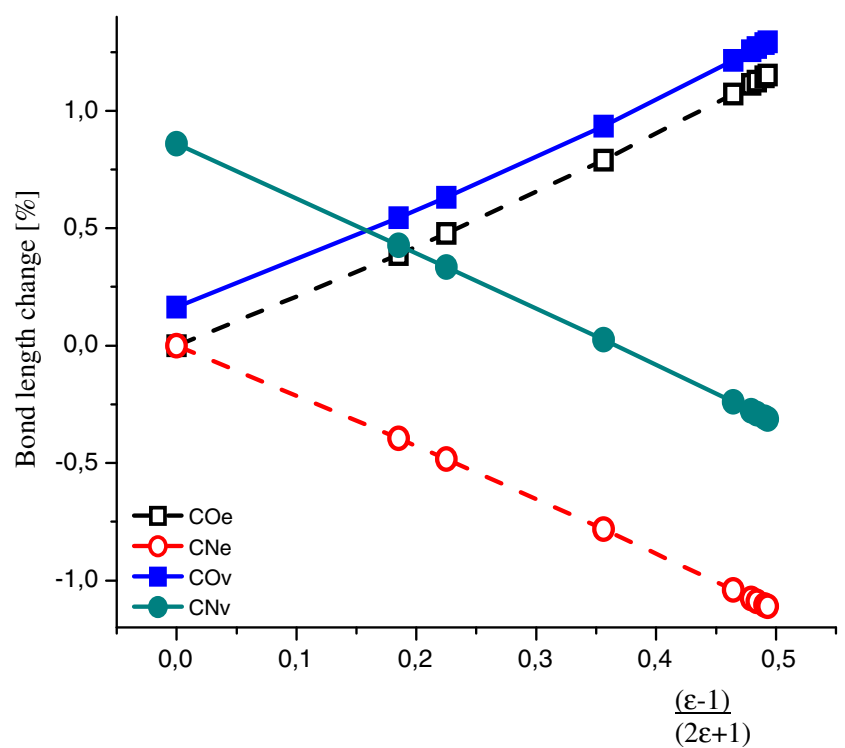

Fig. 3 Solvent dependence of equilibrium and anharmonically averaged formamide $\mathrm{C}=\mathrm{O}$ and $\mathrm{C}-\mathrm{N}$ bond lengths relative to equilibrium gas phase values calculated at B3LYP/pc-2 level (in \%)

First, the percentage changes of $\mathrm{C}=\mathrm{O}$ and $\mathrm{C}-\mathrm{N}$ bonds at equilibrium in the gas phase, and rovibrationally averaged by the VPT2 method were plotted as a function of medium polarity in Fig. 3. These results show that both bond lengths change linearly in response to medium polarity. Thus, in comparison to vacuum, the $\mathrm{C}=\mathrm{O}$ bond is longer by about + $1 \%$, and the $\mathrm{C}-\mathrm{N}$ bond is shorter by about $-1 \%$ in formamide solution. It is also apparent that the equilibrium $\mathrm{C}=\mathrm{O}$ bond length and rovibrationally averaged length are essentially similar (the corresponding lines are parallel and separated by only $0.2 \%$ of the initial equilibrium structure in vacuum). On the other hand, the corresponding $\mathrm{C}-\mathrm{N}$ bond lengths are fairly different (well separated lines are observed in Fig. 3). Obviously, the elongation of the $\mathrm{C}=\mathrm{O}$ and shortening of the $\mathrm{C}-\mathrm{N}$ bond in response to solvent polarity reflects the increased contribution of the more polar structure B due to amide resonance (Fig. 4). We also noted that the equilibrium and rovibrationally averaged structural parameters obtained for the isolated formamide molecule reproduces experimental gas phase data fairly well $[35,36]$. Similarly, the agreement between our rovibrational averaged formamide structural parameters calculated in formamide solution is close to recent $a b$ initio moleculardynamic data for liquid formamide reported by Tsuchida [37].

We conclude that the VPT2 method in combination with the PCM model is able to correctly predict changes of rovibrationally averaged structural parameters in response to solvent polarity. On the other hand, calculated frequencies are second derivatives of total energy of the system and changes of atomic positions. Thus, all minute errors in geometry or energy calculations are seen as if through a "magnifying glass" in the calculated anharmonic frequencies. Therefore, in the case of formamide, some anharmonic frequencies behave erratically upon increasing solvent polarity. A similar sensitivity of calculated magnetic parameters, for example, the accuracy of nuclear isotropic shieldings, is observed upon application of correlationconsistent or polarization-consistent basis set [18, 34]. Another reason for the unphysical changes in anharmonic $\mathrm{CN}$ stretch vibration with solvent polarity could be Fermi resonance with a $2 v_{11}$ overtone.

\section{Conclusions}

In this paper, for the first time, we showed the convergence of formamide harmonic and anharmonic (calculated using the VPT2 method) frequencies toward their corresponding B3LYP/pc-n CBS limits in the gas phase and in solution. A systematic study of the basis set convergence was carried out. From our results, the following conclusions can be drawn:

1. In the case of polarization consistent basis sets, starting from pc-2, a regular and systematic convergence (leading mainly to a decrease in wavenumber) of both harmonic and anharmonic frequencies in the gas phase and solution is observed. In fact, harmonic and anharmonic wavenumbers practically converge for pc2 , and sometimes for pc-3, basis sets, making calculations with larger basis sets unnecessary. Only in the case of anharmonic $\mathrm{C}-\mathrm{N}$ stretching mode (and contrary to the corresponding harmonic counterpart), is a very strong dependence on Jensen's basis set size observed, and the corresponding CBS limit underestimates the experimental value. Thus, this mode is difficult to predict correctly by VPT2 combined with B3LYP.

2. Increasing the size and quality (flexibility) of Pople basis set leads to irregular and non-monotonic changes in formamide harmonic and anharmonic frequencies. However, the frequencies calculated with fairly large 6$311++\mathrm{G}^{* *}$ and $6-311++\mathrm{G}(3 \mathrm{df}, 2 \mathrm{pd})$ basis sets approach the CBS limit observed for pc-n basis sets.

3. The most important and diagnostic amide mode $(\mathrm{C}=\mathrm{O}$ stretching), relevant to studies on amides and peptides, is fairly well predicted by VPT2 method, both in the

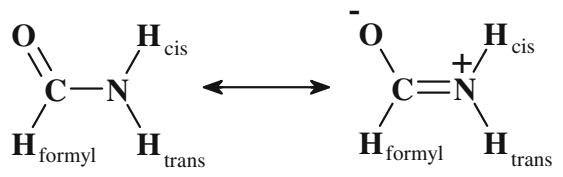

A

B

Fig. 4 Amidic resonance of formamide 
gas phase and in solvents of low, medium and high polarity.

4. The quality of anharmonic frequencies is inferior to simple harmonic frequency scaling procedures (RMS in case of B3LYP/6-31G* calculations is $53 \mathrm{vs} 19 \mathrm{~cm}^{-1}$, and for B3LYP/CBS results is $59 \mathrm{vs} 23 \mathrm{~cm}^{-1}$ ). This fact is important for studies on peptide systems.

5. Solvent dependence of some low frequency anharmonic amide modes of formamide (e.g., CN stretch, $\mathrm{NH}_{2}$ scissoring, and $\mathrm{NH}_{2}$ in plane bend) is incorrect. The VPT2 method reproduces some formamide frequencies in solution in an irregular way, probably due to the presence of severe anharmonicity and Fermi resonance.

Acknowledgments A.B. is a recipient of a $\mathrm{PhD}$ fellowship from a project funded by the European Social Fund. Calculations were carried out in Wroclaw Centre for Networking and Supercomputing (http://www.wcss.wroc.pl), and in the Academic Computer Centre CYFRONET, AGH, Kraków, grant MEiN/SGI3700/UOpolski/063/ 2006. One of us (T.K.) was supported by grant 10/WCH/2010-S.

Open Access This article is distributed under the terms of the Creative Commons Attribution Noncommercial License which permits any noncommercial use, distribution, and reproduction in any medium, provided the original author(s) and source are credited.

\section{References}

1. Hehre WJ, Radom L, PvR S, Pople JA (1986) Ab initio molecular orbital theory. Wiley, New York

2. Scott AP, Radom L (1996) Harmonic vibrational frequencies: an evaluation of Hartree-Fock, Möller-Plesset, quadratic configuration interaction, density functional theory, and semiempirical scale factors. J Phys Chem 100:16502-16513

3. Merrick JP, Moran D, Radom L (2007) An evaluation of harmonic vibrational frequency scale factors. J Phys Chem A 111:1168311700

4. Sinha P, Boesch SE, Gu C, Wheeler RA, Wilson AK (2004) Harmonic vibrational frequencies: scaling factors for HF, B3LYP, and MP2 methods in combination with correlation consistent basis sets. J Phys Chem A 108:9213-9217

5. Jensen F (1999) The basis set convergence of the Hartree-Fock energy for $\mathrm{H}_{2}$. J Chem Phys 110:6601-6605

6. Jensen F (2001) Polarization consistent basis sets: principles. J Chem Phys 115:9113-9125

7. Jensen F (2002) Polarization consistent basis sets: II. Estimating the Kohn-Sham basis set limit. J Chem Phys 116:7372-7379

8. Jensen F (2003) Polarization consistent basis sets. IV. The basis set convergence of equilibrium geometries, harmonic vibrational frequencies, and intensities. J Chem Phys 118:2459-2463

9. Jensen F, Helgaker T (2004) Polarization consistent basis sets. V. The elements Si-Cl. J Chem Phys 121:3463-3470

10. Jensen $F$ (2005) The effect of different density functional methods on basis set parameters. Chem Phys Lett 402:510-513

11. Dunning TH Jr (1989) Gaussian basis sets for use in correlated molecular calculations. I. The atoms boron through neon and hydrogen. J Chem Phys 90:1007-1023

12. Dunning TH Jr (2000) A road map for the calculation of molecular binding energies. J Phys Chem A 104:9062-9080
13. Wilson A, van Mourik T, Dunning TH Jr (1996) Gaussian basis sets for use in correlated molecular calculations. VI. Sextuple zeta correlation consistent basis sets for boron through neon. J Mol Struct THEOCHEM 388:339-349

14. Kendall RA, Dunning TH Jr, Harrison RJ (1992) Electron affinities of the first-row atoms revisited. Systematic basis sets and wave functions. J Chem Phys 96:6796-9806

15. Helgaker T, Klopper W, Koch H, Noga J (1997) Basis-set convergence of correlated calculations on water. J Chem Phys 106:9639-9646

16. Petersson GA, Al-Laham MA (1991) A complete basis set model chemistry. II. Open-shell systems and the total energies of the first-row atoms. J Chem Phys 94:6081-6090

17. Feyereisen MW, Feller D, Dixon DA (1996) Hydrogen bond energy of the water dimer. J Phys Chem 100:2993-2997

18. Kupka T, Lim C (2007) Polarization-consistent vs correlationconsistent basis sets in predicting molecular and spectroscopic properties. J Phys Chem A 111:1927-1932

19. Wang NX, Wilson AK (2003) Effects of basis set choice upon the atomization energy of the second-row compounds $\mathrm{SO} 2, \mathrm{CCl}$, and ClO2 for B3LYP and B3PW91. J Phys Chem A 107:6720-6724

20. Campos CT, Jorge FE, Silva TP, Coppo MR (2010) Basis set convergence on optical rotation DFT calculations. Chem Phys Lett 494:170-173

21. Boese AD, Klopper W, Martin JML (2005) Assessment of various density functionals and basis sets for the calculation of molecular anharmonic force fields. Int J Quantum Chem 104:830-845

22. Cappelli C, Monti S, Scalmani G, Barone V (2010) On the calculation of vibrational frequencies for molecules in solution beyond the harmonic approximation. J Chem Theor Comput 6:1660-1669

23. Bounouar M, Scheurer C (2008) The impact of approximate VSCF schemes and curvilinear coordinates on the anharmonic vibrational frequencies of formamide and thioformamide. Chem Phys 347:194-207

24. Bour P, Bednarova L (1995) Anharmonic force field of formamide. A computational study. J Phys Chem 99:5961-5966

25. Carbonniere P, Dargelos A, Ciofini I, Adamo C, Pouchan C (2009) Vibrational analysis of glycine radical: a comparative ab initio static and dynamic study. Phys Chem Chem Phys 11:43754384

26. Barone V (2004) Vibrational zero-point energies and thermodynamic functions beyond the harmonic approximation. J Chem Phys 120:3059-3065

27. Barone V (2005) Anharmonic vibrational properties by a fully automated second-order perturbative approach. J Chem Phys 122:014108(10)

28. McNaughton D, Evans CJ, Lane S, Nielsen CJ (1999) The highresolution FTIR far-infrared spectrum of formamide. J Mol Spectrosc 193:104-117

29. Marigliano ACG, Varetti EL (2002) Self-association of formamide in carbon tetrachloride solutions: an experimental and quantum chemistry vibrational and thermodynamic study. J Phys Chem A 106:1100-1106

30. Frisch MJ, Trucks GW, Schlegel HB, Scuseria GE, Robb MA, Cheeseman JR, Montgomery JA Jr, Vreven T, Kudin KN, Burant JC, Millam JM, Iyengar SS, Tomasi J, Barone V, Mennucci B, Cossi M, Scalmani G, Rega N, Petersson GA, Nakatsuji H, Hada M, Ehara M, Toyota K, Fukuda R, Hasegawa J, Ishida M, Nakajima T, Honda Y, Kitao O, Nakai H, Klene M, Li X, Knox JE, Hratchian HP, Cross JB, Bakken V, Adamo C, Jaramillo J, Gomperts R, Stratmann RE, Yazyev O, Austin AJ, Cammi R, Pomelli C, Ochterski JW, Ayala PY, Morokuma K, Voth GA, Salvador P, Dannenberg JJ, Zakrzewski VG, Dapprich S, Daniels AD, Strain MC, Farkas O, Malick DK, Rabuck AD, Raghavachari K, Foresman JB, Ortiz JV, Cui Q, Baboul AG, Clifford S, 
Cioslowski J, Stefanov BB, Liu G, Liashenko A, Piskorz P, Komaromi I, Martin R. L, Fox DJ (2009) Gaussian 09, Revision A.02. Gaussian Inc, Wallingford, CT

31. EMSL basis set exchange. https://bse.pnl.gov/bse/portal

32. Miertus S, Scrocco E, Tomasi J (1981) Electrostatic interaction of a solute with a continuum. A direct utilization of $a b$ initio molecular potentials for the prevision of solvent effects. Chem Phys 55:117-129

33. Buczek A, Kupka T, Broda MA (2010) Extrapolation of water and formaldehyde harmonic and anharmonic frequencies to the B3LYP/CBS limit using the polarization consistent basis sets. J Mol Model. doi: 10.1007/s00894-010-0913-3
34. Kupka T, Stachów M, Nieradka M, Kaminsky J, Pluta T (2010) Convergence of nuclear magnetic shieldings in the Kohn-Sham limit for several small molecules. J Chem Theor Comput 6:15801589

35. Hirota E, Sugisaki R, Nielsen CJ, Sorensen GO (1974) Molecular structure and internal motion of formamide from microwave spectrum. J Mol Spectrosc 49:251-267

36. Kitano M, Kuchitsu K (1974) Molecular structure of formamide as studied by gas electron diffraction. Bull Chem Soc Jpn 47:6772

37. Tsuchida E (2004) Ab initio molecular-dynamics study of liquid formamide. J Chem Phys 121:4740-4746 